\title{
LINGÜÍSTICA Y LENGUAJE LITERARIO
}

\author{
ÁNGEL HERRERO \\ (Universidad de Alicante)
}

\begin{abstract}
RESUMEN
This article deals with some revelant aspects of the linguistics of literature (or 'linguistics of writing') such as it has been discussed during the last two decades as a result of the jakobsonian metapoetics postulate. Two contributions are cmphasized, both previous to the famous Congress of Indiana but divulged nevertheless a few years later: the Saussure's anagrams and the peircean iconic theory.
\end{abstract}

La relación de la linguística con el conjunto de las disciplinas humanísticas, y particularmente con la fillología, ha sufrido en las últimas tres décadas una transformación que ha llegado a ser calificada de «dramática» por su intensidad, desde la abierta discrepancia de intereses de los años 60 hasta el momento actual en el que, según diagnosticaba Sydney M. Lamb siendo aún presidente de la Asociación de Lingüística de E.E.U.U. y Canadá, «ya no hay fronteras que separen la linguística, de la poética, de la retórica y del estudio de la literatura en gencral» (1984: 5). Esta transformación, que vuelve a postular el carácter originario de la literatura en el desarrollo onto y filogenético de la actividad lingüística y del pensamiento, es la que se refleja en la llamada lingiiística de la escritura, o linguíística del confacto, en el sentido programático con que la define Mary Louise Pratt (Fabb 1987: 67) o, de forma más simple y tal vez más clara, lingiḯstica de la literatura, linguísticas que pretenden conjurar a un tiempo el csoterismo formal de la lingüística estructural y la vaguedad de postulados de la fïlología positivista.

La nueva corriente responde a la confluencia de varias aportaciones teóricas de extraordinaria importancia, cuyo alcance hace previsible la influencia del nuevo paradigma en aspectos fundamentales de la concepción del lenguaje en general y del lenguaje literario en particular. En estas páginas voy a señalar algunas de esas confluencias y de estos aspectos sin poner de mi parte, interesada 
desde hace ya bastantes años en la cuestión', más que la evaluación que va de suyo en toda muestra selectiva y algún argumento añadido a los que aportaré desde voces sin duda más autorizadas. Sin embargo, es necesario advertir que la confrontación entre literatura, filología y lingüística rebasa con mucho el marco de ninguna lendencia particular, y puede detectarse en diversos campos de estudio con intereses muy distintos (Fisiak 1990; Bernstein 1994a, 1994b; Klein 1995), y de forma particularmente significativa en el de la educación universitaria, en torno a cuyos curricula de Lingüística y Literatura se han venido organizando la mayoría de los encuentros de los últimos años (Lemke 1984; Baker 1991).

Como se sabe, el punto de partida, la aportación programática de mayor eco en la lingüística —que apenas si estaba comenzando a ser generativa, y no era aún sistémica - apuntando explícitamente en la dirección de una colaboración estrecha entre lingüistica y poética, fue la conferencia de clausura del Congreso que bajo este mismo título se celebró en Indiana en 1958, conferencia esperadísima a cargo de Roman Jakobson, y que ha sido glosada innumerables veces, también en nuestro país (Lázaro 1976). Los casi treinta años que transcurrirán hasta el Congreso sobre La lingüística de la Escritura: debates entre lengua y literatura celebrado en Strathclyde en 1986, con el objeto explícito de evaluar y reorientar el programa de Jakobson, representan al mismo tiempo la crítica y la generalización de algunos de los principios de la lingüística de la literatura inspirada en aquel programa.

En un ambiente intelectual más próximo al formalismo estructural que al funcionalismo praguense en el que sin embargo había tenido un papel tan signilicativo (con la elaboración, junto a Tinianov, en 1928, de un manifiesto en pro de la consideración contextual del arte verbal frente a la estricta formalización), Jakobson defendió en aquella conferencia la naturaleza lingüística de la semiosis introversiva verbal, que llamó función poética; con ello se olrecía un criterio fundamental para la poética linguística - el famoso principio de proyección-, pero la lingüística en sí misma, esto es, la concepción del lenguaje de raigambre saussureana, no sólo no se veía afectada sino aparentemente corroborada. El congreso de 1986 mostró una situación prácticamente inversa: un nuevo principio general (la diferiencia ${ }^{2}$ o diferancia), detectado ejemplarmente en el discurso literario, se aplica a la concepción del lenguaje en busca de una nueva lingüística - por construir-. Pero sería tan erróneo limitar las contribuciones de Jakobson a su poética (en relación con ella

Desde 1977, al menos. Vid. A. Herrero «César Vallejo: poética del habla», en Revisła de Occidente, 19, mayo 1977, pp. 60-64; «La caja negra de Larra: retórica de una deconstrucción», Anales de la Universidad de Alicante, 2, 1985, pp. 75-90; Signo/Texto. De gramática a retórica. Alicante, Universidad, 1986; Semiótica y creatividad. La lógica abdactiva. Madrid, Palas Atenea, 1988.

Creo más apropiado emplear este neologismo que no otros recursos gráficos — con la 'e' invertida o mayúscula -, porque evoca inmediatamente en el hispanohablante la imagen de lo diferido, frente a la de lo diferente; también 'diferancia' podría servir, aunque como feo galicismo. 
está el papel de la marca y del tiempo como valores lingüísticos), como suponer que la lingüística de la literatura o de la escritura es obra de quien, sin duda, despertó en el Congreso de 1986 mayor número de adhesiones, Jacques Derrida. Aunque en este congreso Derrida afirma que «De la Gramatología era al mismo tiempo un programa para una linguística de la escritura (...) y una problematización filosófica de la imposibilidad o de la posibilidad limitada de este programa» (Fabb 1987: 260), lo cierto es que el programa se ha venido desarrollando de forma dispersa, y a veces al margen de toda criterio deconstructivista.

Las conferencias de 1986 vinieron sin embargo a hacer explícita una consecuencia de aquella incorporación 'funcional' de la poética a la lingüística defendida por Jakobson en 1958, cosecuencia que éste en cierto modo obvió a expensas de la consideración de la función poética como una función más, pero al mismo tiempo específica: el hecho de que no hay discontinuidad funcional entre la comunicación cotidiana y la literatura, como desde 1971, y precisamente en otro congreso organizado en Oxford sobre Estilo literario por S. Chatman, venía demostrando M. A. K. Halliday (1971: 364). En 1980, Stanley Fish publica $I s$ There a Text in This Class?, en el que intenta demostrar también que toda noción de lenguaje normal respecto al que pueda definirse un lenguaje literario en función de ciertos rasgos específicos resulta inútil. Las intervenciones en el Congreso de 1986 fueron prácticamente unánimes en este punto, y las de John Hollander y Mary Louise Pratt especialmente radicales. Esta última había ya comenzado su tesis doctoral de 1983 «Toward a Speech Theory of Literary Discourse» - parcialmente contestada por los teóricos de los actos de hablaseñalando que

the contribution of these schools (se refería al formalismo y al estructuralismo en general) to our understanding of specific literary texts has been enormous and valuable, but its point of departure, the poetic/no-poetic opposition in its various guises, has indeed been, as Roger Fowler says, 'one of the greatest sources of confusion and error in poetics' (Pratt 1983: 2).

En su artículo «Linguistics, Poetics, and the Literary Genres», de 1984, Edward Stankiewicz hace suya la crítica generalizada a la inclusión de la función poética en los esquemas funcionales como una función distintiva:

The 'poetic function' -escribe- is by no means conmensurate with the other functions of language (...) Poetry may manipulate the sources of language, but it remains a function of parole, even though some traditions of poctry have at various times created special poetic languages (...) The use of such languages or of distinctly poetic forms is neither a necessary nor a sulficient condition for the construction of literary texts. 
y terminaba diagnosticando que «jakobson's proyection rule is, furthermore, an overgeneralization, yet is not general enough» (Stankiewicz 1984: 163).

La reinterpretación de la función jakobsoniana se produjo así sobre dos grandes ejes: por un lado, en un sentido verdaderamente funcional (o pragmático), como 'orientación no hacia el mensaje sino hacia el discurso' -y con él hacia los interlocutores-, como propuso Geoffrey Leech (Fabb 1987: 94); esto significa que la recursividad de unidades paradigmáticas como contenido del principio de proyección jakobsoniano se sustituye por la recursividad interpretativa, porque —como escribe Widdowson (Fabb 1987: 255) - «la significación del poema es función de la experiencia real de la interpretación, de la recurrencia de la atención, de la revisión de las primeras impresiones, de la activación de los significados potenciales inherentes a las formas lingüísticas, del acto analítico de desbrozar lo que pueden significar los signos lingüísticos dentro del patrón dual de la sintaxis y la prosodia».

Esta rectificación del contenido del principio de proyección suponía a su vez una importante reconsideración del principio de iconicidad, que explica el autotelismo de la obra entendido no como fin-en-sí-misma, como satisfacción colmada, como resultado acabado de una proyección de equivalencias, sino, al contrario, como escenario de nuevos posibles textos, como llamada a su repetición creadora. En este sentido, el debate sobre el carácter convencional de lo icónico es sin duda uno de los fenómenos actuales más significativos en el campo de la teoría semiótica y lingüística, a pesar de la influencia, tan extensa como negativa desde mi punto de vista, de la crítica radicalmente convencionalista de Umberto $\operatorname{Eco}^{3}$ a toda teoría automostrativa o esencialista —en el sentido de Genette (1993: 15) — de la icogénesis peirceana. El tema, apasionante, ha dado lugar ya a varias monografías cuyo interés está fuera de toda duda (Bouissac, Herzfeld y Posner 1986; Simone 1994). Nuestro punto de vista coincide prácticamente con el defendido por J. Ransdell (1986): que la teoría semiótica de Peirce nos libra de la tiranía del significante convencional, de la concepción instrumental y vinculante del significante fónico, y nos devuelve la imagen del signo como hacedor de interpretantes, no como mero transmisor de signilicados.

La lingǘstica de la escritura mantiene, y esto es fundamental, el carácter esencialista de la poética jakobsoniana - véase la defensa de este punto de vista en la intervención de P. Kiparsky-, pero desde la perspectiva de una nueva concepción del lenguaje como sistema abierto dinámico, tal y como propuso J. L. Lemke desde el análisis de los procesos instruccionales (Lemke 1984) y M. A. K. Halliday

U. Eco ha mantenido su posición convencionalista -en el sentido de entender la iconicidad como pertinentización o selección culturalmente decidida de un conjunto de rasgos perceptivos del objeto-desde La estrucfura ausente, de 1972. Entre sus reflexiones porteriores sobre la iconicidad, véase «Pour une reformulation du concept de signe iconique», Communications, 29, París, Seuil, 1978, pp. 141-191, y «Mirrors» en Bouissac, Herzfeld y Posner (eds.), 1986, pp. 215-238. Para una crítica al punto de vista de Eco, vid. Paul Bouissac, «lconicity and Pertinence», en Bouissac, Herzield y Posnel (cds.), 1986, pp. 193-213. 
reivindicó en su intervención (Fabb 1987: 150). Paul Kiparsky explicaba el relativo fracaso del programa jakobsoniano diciendo que

el error se encuentra en lo inadecuado de las teorías del lenguaje y de la comunicación que le sirvieron de base; en sí mismo, el programa es válido y ha proporcionado interesantes resultados siempre que ha sido aplicado con la lingüística y la pragmática apropiadas (193).

La nueva lingüística, en palabras de Jonathan Culler, trata de «recomponer la empresa (de la linguística estructural) intentando situar lo marginal en el centro» (Fabb 1987: 184); esta inversión se convierte en programática:

Una lingüústica de la escritura (...) trataría de invertir la relación habitual entre signos discretos, ya codificados, y el material normalmente juzgado irrelcvante excepto como medio de manifestación. Consideraría los signos discretos como casos especiales de una resonancia general (...) La tarea de la lingüística ha sido dividir lo significativo de lo no significativo, excluyendo esto último de la lingüística, pero si esta región fronteriza es central en el lenguaje y en su funcionamiento, entonces se debe revisar su geografía, y el carácter incierto de las resonancias, la materialidad problemática del lenguaje que puede o no ser portadora de significado y producir o no efectos, deben situarse en el centro de nuestros intereses.

Declaración de intenciones que el propio Culler reconoce incierta: «Tal vez no sea posible construir una lingüística sobre esta base (...) pero parece un valioso experimento, de cuyo fracaso podríamos aprender casi tanto como de su éxito».

Es muy significativo que la evolución teórica del paradigma estructural saussureano a través de la psicosistemática, y la más actual del paradigma generativo, a través de las propuestas de S. Abney, avancen en esta reconversión de lo marginal en nuevo centro de intereses y de análisis (en el significante léxico, por la generalización de la noción de significancia; en el significante sintáctico, con la nuclearización de los especificadores, esto es, de los indicadores deícticos de los sintagmas nominales y verbales), y sin duda es el alcance mismo de la renovación lingǘstica lo que nos hace sentir más su utopía (como M. Louise Pratt subrayó) que su inmediata aplicación. No podemos apenas calibrar aún lo que significará —más allá de la adecuación descriptiva, que parece acertada- la sustitución de la noción exocéntrica de la oración gramatical como expresión de una predicación lógica, por otra en la que lo nuclear es la gramaticalización de un contexto; un momento histórico en el que la noción clave de la gramática sufre tal transformación es un momento sometido necesariamente a otros muchos cambios: la noción misma de pensamiento, la de verdad, la de acción y licción, no pueden ser insensibles a esta descentralización del logos que también las constituía. Y la filosofía, desde el pragmatismo (y muy especialmente cn su versión ortegiana, tematizando la circunstancia) hasta al menos Foucault, que escribió páginas admirables sobre El pensamiento del afuera, parece estar 
recorriendo un camino similar. El discurso, como nuevo objeto de estudio, y aún con todas las cautelas que un objeto borroso por su aplitud debe acarrear (Widdowson 1995), representa esa exterioridad del lenguaje antes velada y ahora sin embargo reveladora de aspectos insólitos de la producción lingüística y del lenguajc en general.

Yo diría que la crítica y la solución de la poética jakobsoniana que, como vemos, implican nada menos que una nueva lingüística, se venía produciendo ya en ese escenario teórico que la vio nacer, y que no se limitaba a la lingüística: el escenario de la semiótica peirceana, de la que Jakobson fue el más destacado difusor en aquellos años, y a la que Derrida debe mucho más de lo que él mismo declaró en su Gramatología: nociones como las de huella y diferancia no son, descle mi punto de vista, sino etiquetas para exponer, de forma indudablemente más plástica, la incidencia del interpretante peirceano -esa noción revolucionaria todavía-. La semiótica de Peirce (y, como después señalaré, la del 'otro Saussure') es la semiótica inspiradora de la lingüística de la escritura, como lo es de la semítica de los cambios estables y de las singularidades de René Thom, aplicada por Jean Petitot a la fonología y a la sintaxis. El diagrama de equivalencias que se desprende de los textos dominados por la función poética, tal y como Jakobson lo ideó y lo practicó en numerosísimos análisis, es sin duda deudor de los diagramas icónicos de Peirce. Sin embargo, los diagramas peirceanos son sólo uno de los tres tipos o dimensiones icónicas señalados por el sabio americano: la experiencia lectora de un poema no es la que se experimenta ante un diagrama habitual, por ejemplo ante el mapa de una ciudad, porque en éste el lector asume el isomorfismo entre las partes constitutivas del signans y las partes constitutivas del signatum; tal es el efecto que se produce con el orden sintáctico habitual (Simone 1994). En el poema, en cambio, se experimenta más la emoción propia de una imagen o una metáfora, los otros iconos señalados por Peirce, que someten las relaciones diagramáticas normales a un proceso de reiconización ${ }^{4}$.

La crítica a la función poética jakobsoniana empezó a producirse por ello desde la semiótica peirceana; me quiero referir especialmente a la crítica llevada a cabo con extraordinario tesón y rigor por Michael Shapiro — no citado por ninguno de los conferenciantes de 1986-. En numerosas publicaciones desde su Asymmetry, de 1976, Shapiro, que ha propuesto entender el interpretante peirceano como valor lingüístico (Herrero 1988: 160), ha venido defendiendo el carácter asimétrico del interpretante-marca, frente al equilibrio de marcas que proponía el principio de equivalencia de Jakobson, y con ello la necesidad de proyectar extroversivamente este principio de equivalencia entendiéndolo como un proceso que afecta, en un orden abierto, a todas las dimensiones del signo peirceano:

Adoptamos el término de Anna Giacalone Ramat, «lconicity in Grammaticalization Processes», en R. Simone (ed.), 1994, pp. 119-139. 
The same asymmetry between encoder and decoder that informs the ordinary use of language also constitutes the core of the poetic situation, with its necessary and insistent elicitation of a series of interpretants, in a teleological movement that attempts to match interpretation with intentionality. The self-referential component of the artistic sign, the one that accounts for the measure of parallelism and recurrence - the Secondness- to be found in a work of verbal art, is ultimately transcended in the hierarchy of semeiosis by representation or Thirdness. In goig beyond the limits of iconicity or self-referenciality, poetry remains foundationally true to its own nature as symbolic art (1980: 110).

La sustitución del criterio de orientación al mensaje —como definición de la función poética- por el de orientación al discurso, que antes apuntábamos como propuesta de la lingüística de la escritura, puede reconducirse así y, más allá del interés teórico en una u otra zona de la producción verbal, ser entendida como un hecho que la propia semiosis poética produce.

También las soluciones ofrecidas por la linguística del contacto (Pratt), que Derrida consideró como la lingüística de la escritura más adecuada por su carácter dinámico y abierto, descentralizado, eran soluciones ya aportadas desde la semiótica, sobre todo por I. Lotman, cuyo artículo de 1977 «The Dinamyc Model of Scmiotic System» exponía prácticamente los mismos criterios de descentralización del análisis semiótico que propusieron en 1986 Mary Louise Pratt y M. A. K. Halliday.

Lo verdaderamente importante del programa de lingüística de la literatura desprendido del congreso de 1986 no está por ello, desde mi punto de vista, ni en la crítica ni en la solución a la cuestión de la función poética o de la literariedad, sino en haber hecho de esta cuestión - y en la medida en que el lenguaje vive en esos textos de forma plena- el catalizador de una nueva perspectiva lingüística general; dicho de otro modo, en haber avanzado en la consideración de la genuina iconicidad que promueve el lenguaje, y que la literatura convierte en espectáculo.

Paradójicamente, la linguística recorría así el camino mismo de la filología, pero sin abandonar su empeño teórico. Y parece evidente que esa coincidencia no es puramente genealógica; si observamos la lingüística como una semiótica, las palabras con que abría Cesare Segre su Semiótica filológica nos resultarán clarividentes: «La actitud filológica puede salvar la semiótica del narcisismo de la palabra, de la embriaguez de fugas sin retorno» (1990: 16). La concreción filológica que todos hemos admirado en numerosos trabajos de Jakobson (pero también de la estilística) vuelve de nuevo a ser central precisamente porque el sistema se revela como un sistema de concreciones ininterrumpidas, que 'espesan' en dilerentes formas. En un campo próximo como es el de la retórica, hace tiempo que se ha aceptado que «philology —enriched by the modern advances in linguistics, cultural history, etc., and on the other hand continuing its brillant tradition- is indispensable to rhetorical analysis», siendo la falta de detalle filológico lo que ha limitado la investigación retórica moderna (Valesio 1980: 
55). La Teoría de la estructura retórica (Mann 1992) pretende sistematizar esta integración de retórica, filología y lingüística. En nuestra tradición, la adicción metodológica al detalle filológico fue representada sin duda por la estilística, y es sintomático que sea en Estados Unidos donde ha experimentado en las últimas décadas su mayor auge (Brahim 1991; Dehennin 1994).

Muchos de los mejores trabajos deconstructivos de la lingǘrstica de la escritura versan sobre textos literarios, y tratan especialmente su valor figural para reivindicar la propiedad precisamente lingüística de las figuras (Hollander, Levin, Kiparsky, Moravcisk...), como fenómenos criptogramaticales, para emplear el término acuñado por M. A. K. Halliday. Pero es que «así configura la lengua el orden natural: mediante los patrones inconscientes, criptolípicos, de la gramática, que crean su propio orden de la realidad independientemente de aquello para cuya descripción se los pueda estar utilizando» (Fabb 1987: 153). La cuestión, de nuevo, está en precisar la naturaleza de esos patrones inconscientes. Derrida ha hecho suyos los criterios de la semántica de prototipos, para superar, mediante la noción de diferiencia, la oposición entre diferencia de tipo y diferencia de grado (Fabb 1987: 265). Pero las propuestas son hasta ahora más crípticas que gramaticales. Y he aquí la nueva paradoja: que intentando superar la noción de distintividad y discrecionalidad del signo saussureano, sometiéndolo a una crítica que pueda dar cuenta de su génesis y de sus veleidades, se haya llegado a reivindicar al mismo Saussure (porque no hubo «otro Saussure») que poco antes de dictar el Cours estaba entregado al estudio de una criptogramática de la poesía antigua, elaborada según él de modo anagramático, desde la inventio misma.

Anagramas saussureanos, iconicidad peirceana, he aquí las estrellas del debate. La exhumación de los Cahiers de anagramas de Saussure, especialmente a cargo de Jean Starobinski (1971), ha tenido como efecto (además de la elaboración de poéticas específicas, como la hipogramática de Michael Rilfaterre, o de la comprensión de ciertos poemas o poetas cuyo recurso al anagramatismno había pasado desapercibido ${ }^{5}$ ) una deconstrucción del Saussure canónico que aún no ha terminado, pero que constituye uno de los horizontes de la nueva lingüística. Como escribía Jonathan Culler:

Si la concepción de Ferdinad de Saussure del lenguaje como sistema de signos se puede ver como la base de esta primera linguística (la fonocéntrica), la segunda lingüística se enfrentará desde el principio a los problemas que obsesionaban al 'otro Saussure', como se le ha llamado, el Saussure de los anagramas (1987: 185).

Pero para que esta lingüística pueda avanzar en su alcance teórico y en su capacidad explicativa es preciso realizar aún la síntesis entre esta hipótesis de

Vid. A. Herrero «La sibilación escrita. Anagramatismo en la poesía de Antonio Machado», Bulletin Hispanique, 98, 1, 1996, pp. 205-219. 
Saussure y el resto de hipótesis o nociones que estos años de revisión y preparación de un nuevo paradigma han ido despejando: la noción de iconicidad peirceana, la de interpretante, la de significancia, la de singularidad. La lingüística de la escritura sólo podría construirse, desde mi punto de vista, como una lingüística basada en la integración teórica de estas cuestiones.

\section{Referencias bibliográficas}

BAKER, C. \& LUKE, A. (eds.) (1991): Towards a Critical Sociology of Reading Pedagogy: Papers of the XII World Congress on Reading. Amsterdam, John Benjamin Pub. Co.

BERNSTEIN, C. G. (1994): «Language and Literature in Context», The SECOL Review, I8, 1, pp. 45-61.

BERNSTEIN, C. G. (ed.) (1994): The Text \& Beyond: Essays in Literary Linguistics. The Univ. of Alabama Press.

BOUISSAC, P. \& HERZFELD, M. \& POSNER, R. (eds.) (1986): Iconicity. Essctys on the Nature of Culture. Tübingen, Stauffenburg Verlag.

DEHENNIN, E. \& HAVERTAKE, H. (eds.) (1994): Linguiística y Estilística de textos. Amsterdam, Atlanta.

FABB, N. \& ATTRIDGE, D. \& DURANT, A. (comp.) (1987): The linguistics of writing. Manchester Univ. Press. Edición española Lingüística de la escritura. Madrid, Visor, 1989.

FISIAK, J. (cd.)(1990): Historical Linguistics and Philology. Berlin, Walter de Gruyter \& Co.

FORASTIERI, E. \& GUINNESS, G. \& LÓPEZ MORALES, H. (eds.) (1980):

On Text and Context. Methodological Approaches to the Contexts of Literature. Puerto Rico, Universidad.

GENETTE, G. (1991): Ficción y Dicción. Barcelona, Lumen, ed. esp. 1993.

HALLIDAY, M. A. K. (1971): «Linguistic function and literary style: an inquiry into William Golding's The Inheritors», en Chatman, S. (ed.): Literary Style: A Symposium, Oxford, pp. 330-365.

HERRERO, A. (1988): Semiótica y creatividad. La lógica abductiva. Madrid, Palas.

KLEIN, W. (1995): «Literaturwissenschaft, Linguistik, LiLi», en Zeitschrift für Literaturwissenschaft und Linguistik (LiLi), 25, 100, pp. 1-10.

LAMB, S. M. (1984): «On the Aims of Linguistics», en James E. Copeland (ed.): New Directions in Linguistics and Semiotics. Amsterdam, John Benjamin Pub. Co., pp. $1-11$.

LAZARO CARRETER, F. (1976): «La lingüística norteamericana y los estudios literarios en la década 1958-1968», y «¿Es poética la función poética?», en Estudios de poética (La obra en sí). Madrid, Taurus, pp. 31-49 y 63-73.

LEMKE, J. L. (1984): Semiotics and Education. Toronto, Toronto Semiotic Circle. 
MANN, W.C. \& THOMPSON, S. (eds.) (1992): Discourse Description: Diverse Linguistic Analyses of a Fund-Raising-Text. Amsterdam, John Benjamin Pub. Co.

MOUSSABIR, B. (1991): Linguistic Models and the Literary Text: Stylistics and Poetics in America from the Fifties to the Eighties. University Microfilms International, Ann Arbor, Michigan.

PRATT, M. L. (1983): Toward a Speech Theory of Literary Discourse. Stanford Univ. UMI, Ann Arbor, Michigan.

RIFFATERRE, M. (1978): Semiotics of Poetry. Londres, Methuen.

SEGRE, C. (1990): Semiótica filológica. Murcia, Sec. Pub. Universidad.

SHAPIRO, M. (1976): Asymmetry. An Inquiry into the Linguistic Structure of Poetry. New York, North Holland Pub.Co.

SHAPIRO, M. (1982): «Remarks on the nature of the autotelic sign» en Heidi Byrnes (ed.): Contemporary Perceptions of Language: Interdisciplinary Dimensions. Georgetown Univ. Press.

SIMONE, R. (ed.) (1994): Iconicity in Language. Amsterdam, John Benjamin Pub. Co.

STANKIEWICZ, E. (1984): «Linguistics, Poetics, and the Literary Genres», en James E. Copeland (ed.): New Directions in Linguistics and Semiotics. Amsterdam, John Benjamin Pub. Co., pp. 155-176.

VALESIO, P. (1980): «The analysis of texts and the critique of ideology», en Forastieri (ed.), pp. 55-74.

WIDDOWSON, H. G. (1995): «Discourse Analysis: A Critical View», en Language and Literature, 4, 3, p. 157. 